\title{
Erratum to: Antimicrobials: a global alliance for optimizing their rational use in intra- abdominal infections (AGORA)
}

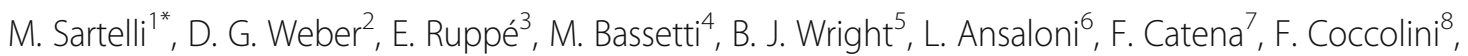
F. M. Abu-Zidan' ${ }^{9}$ R. Coimbra ${ }^{10}$, E. E. Moore ${ }^{11}$, F. A. Moore ${ }^{12}$, R. V. Maier ${ }^{13}$, J. J. De Waele ${ }^{14}$, A. W. Kirkpatrick ${ }^{15}$, E. A. Griffiths ${ }^{16}$, C. Eckmann ${ }^{17}$, A. J. Brink ${ }^{18}$, J. E. Mazuski ${ }^{19}$, A. K. May ${ }^{20}$, R. G. Sawyer ${ }^{21}$, D. Mertz ${ }^{22}$, P. Montravers ${ }^{23}$, A. Kumar ${ }^{24}$, J. A. Roberts ${ }^{25}$, J. L. Vincent ${ }^{26}$, R. R. Watkins ${ }^{27}$, W. Lowman ${ }^{28}$, B. Spellberg ${ }^{29}$, I. J. Abbott ${ }^{30}$, A. K. Adesunkanmi ${ }^{31}$, S. Al-Dahir ${ }^{32}$, M. N. Al-Hasan ${ }^{33}$, F. Agresta ${ }^{34}$, A. A. Althani ${ }^{35}$, S. Ansari ${ }^{36}$, R. Ansumana ${ }^{37}$, G. Augustin ${ }^{38}$, M. Bala ${ }^{39}$, Z. J. Balogh ${ }^{40}$, O. Baraket ${ }^{41}$, A. Bhangu ${ }^{42}$, M. A. Beltrán ${ }^{43}$, M. Bernhard ${ }^{44}$, W. L. Biffl ${ }^{45}$, M. A. Boermeester ${ }^{46}$, S. M. Brecher ${ }^{47}$, J. R. Cherry-Bukowiec ${ }^{48}$, O. R. Buyne ${ }^{49}$, M. A. Cainzos ${ }^{50}$, K. A Cairns ${ }^{51}$, A. Camacho-Ortiz ${ }^{52}$, S. J. Chandy ${ }^{53}$, A. Che Jusoh ${ }^{54}$, A. Chichom-Mefire ${ }^{55}$, C. Colijn ${ }^{56}$, F. Corcione ${ }^{57}$, Y. Cui ${ }^{58}$, D. Curcio ${ }^{59}$, S. Delibegovic ${ }^{60}$, Z. Demetrashvili ${ }^{61}$, B. De Simone ${ }^{62}$, S. Dhingra ${ }^{63}$, J. J. Diaz ${ }^{64}$, I. Di Carlo ${ }^{65}$, A. Dillip ${ }^{66}$, S. Di Saverio ${ }^{67}$, M. P. Doyle ${ }^{68}$, G. Dorj ${ }^{69}$, A. Dogjani ${ }^{70}$, H. Dupont $^{71}$, S. R. Eachempati ${ }^{72}$, M. A. Enani ${ }^{73}$, V. N. Egiev ${ }^{74}$, M. M. Elmangory ${ }^{75}$, P. Ferrada ${ }^{76}$, J. R. Fitchett ${ }^{77}$, G. P. Fraga ${ }^{78}$, N. Guessennd ${ }^{79}$, H. Giamarellou ${ }^{80}$, W. Ghnnam ${ }^{81}$, G. Gkiokas ${ }^{82}$, S. R. Goldberg ${ }^{76}$, C. A. Gomes ${ }^{83}$, H. Gomi' ${ }^{84}$, M. Guzmán-Blanco ${ }^{85}$, M. Haque ${ }^{86}$, S. Hansen ${ }^{87}$, A. Hecker ${ }^{88}$, W. R. Heizmann ${ }^{89}$, T. Herzog ${ }^{90}$, A. M. Hodonou ${ }^{91}$, S. K. Hong ${ }^{92}$, R. Kafka-Ritsch ${ }^{93}$, L. J. Kaplan ${ }^{94}$, G. Kapoor ${ }^{95}$, A. Karamarkovic ${ }^{96}$, M. G. Kees ${ }^{97}$, J. Kenig ${ }^{98}$, R. Kiguba ${ }^{99}$, P. K. Kim ${ }^{100}$, Y. Kluger ${ }^{101}$, V. Khokha ${ }^{102}$, K. Koike ${ }^{103}$, K. Y. Kok ${ }^{104}$, V. Kong ${ }^{105}$, M. C. Knox ${ }^{106}$, K. Inaba ${ }^{107}$, A. Isik ${ }^{108}$, K. Iskandar ${ }^{109}$, R. R. Ivatury ${ }^{76}$, M. Labbate ${ }^{110}$, F. M. Labricciosa ${ }^{111}$, P. F. Laterre ${ }^{112}$, R. Latifi ${ }^{113}$, J. G. Lee ${ }^{114}$, Y. R. Lee ${ }^{115}$, M. Leone ${ }^{116}$, A. Leppaniemi ${ }^{117}$, Y. Li $i^{118}$, S. Y. Liang ${ }^{119}$, T. Loho ${ }^{120}$, M. Maegele ${ }^{121}$, S. Malama ${ }^{122}$, H. E. Marei ${ }^{35}$, I. Martin-Loeches ${ }^{123}$, S. Marwah ${ }^{124}$, A. Massele ${ }^{125}$, M. McFarlane ${ }^{126}$, R. B. Melo ${ }^{127}$, I. Negoi ${ }^{128}$, D. P. Nicolau ${ }^{129}$, C. E. Nord ${ }^{130}$, R. Ofori-Asenso ${ }^{131}$, A. H. Omari ${ }^{132}$, C. A. Ordonez ${ }^{133}$, M. Ouadii ${ }^{134}$, G. A. Pereira Júnior ${ }^{135}$, D. Piazza ${ }^{136}$, G. Pupelis ${ }^{137}$, T. M. Rawson ${ }^{138}$, M. Rems ${ }^{139}$, S. Rizoli ${ }^{140}$, C. Rocha ${ }^{141}$, B. Sakakushev ${ }^{142}$, M. Sanchez-Garcia ${ }^{143}$, N. Sato ${ }^{103}$, H. A. Segovia Lohse ${ }^{144}$, G. Sganga ${ }^{145}$, B. Siribumrungwong ${ }^{146}$, V. G. Shelat ${ }^{147}$, K. Soreide ${ }^{148}$, R. Soto ${ }^{149}$, P. Talving ${ }^{150}$, J. V. Tilsed ${ }^{151}$, J. F. Timsit ${ }^{152}$, G. Trueba ${ }^{153}$, N. T. Trung ${ }^{154}$, J. Ulrych ${ }^{155}$, H. van Goor ${ }^{49}$, A. Vereczkei ${ }^{156}$, R. S. Vohra ${ }^{157}$, I. Wani ${ }^{158}$, W. Uhl ${ }^{90}$, Y. Xiao ${ }^{159}$, K. C. Yuan ${ }^{160}$, S. K. Zachariah ${ }^{161}$, J. R. Zahar ${ }^{162}$, T. L. Zakrison ${ }^{163}$, A. Corcione ${ }^{164}$, R. M. Melotti ${ }^{165}$, C. Viscoli ${ }^{166}$ and P. Viale ${ }^{167}$

\section{Erratum}

The original article [1] contains an error whereby a co-author, Boris Sakakushev has their family name spelt incorrectly as 'Sakakhushev'.

The authors would therefore like it known that the correct spelling of the family name is 'Sakakushev'.

\footnotetext{
* Correspondence: massimosartelli@gmail.com; m.sartelli@virgilio.it ${ }^{1}$ Department of Surgery, Macerata Hospital, Via Santa Lucia 2, 62100 Macerata, Italy

Full list of author information is available at the end of the article
}

\begin{abstract}
Author details
'Department of Surgery, Macerata Hospital, Via Santa Lucia 2, 62100

Macerata, Italy. ${ }^{2}$ Department of Trauma Surgery, Royal Perth Hospital, Perth, Australia. ${ }^{3}$ Genomic Research Laboratory, Geneva University Hospitals, Geneva, Switzerland. ${ }^{4}$ Infectious Diseases Division, Santa Maria Misericordia University Hospital, Udine, Italy. ${ }^{5}$ Department of Emergency Medicine and Surgery, Stony Brook University School of Medicine, Stony Brook, NY, USA. ${ }^{6}$ General Surgery Department, Papa Giovanni XXIII Hospital, Bergamo, Italy. ${ }^{7}$ Department of General, Maggiore Hospital, Parma, Italy. ${ }^{8}$ Department of Surgery, "Infermi" Hospital, Rimini, Italy. ${ }^{9}$ Department of Surgery, College of Medicine and Health Sciences, UAE University, Al-Ain, United Arab Emirates. ${ }^{10}$ Department of Surgery, UC San Diego Medical Center, San Diego, USA. ${ }^{11}$ Department of Surgery, University of Colorado, Denver Health Medical Center, Denver, CO, USA. ${ }^{12}$ Department of Surgery, Division of Acute Care Surgery, and Center for Sepsis and Critical IIIness Research, University of Florida College of Medicine, Gainesville, FL, USA. ${ }^{13}$ Department of Surgery,
\end{abstract}


University of Washington, Seattle, WA, USA. ${ }^{14}$ Department of Critical Care Medicine, Ghent University Hospital, Ghent, Belgium. ${ }^{15}$ General, Acute Care, and Trauma Surgery, Foothills Medical Centre, Calgary, AB, Canada. ${ }^{16}$ General and Upper GI Surgery, Queen Elizabeth Hospital, Birmingham, UK.

${ }^{17}$ Department of General, Visceral, and Thoracic Surgery, Klinikum Peine, Academic Hospital of Medical University Hannover, Peine, Germany.

${ }^{18}$ Department of Clinical microbiology, Ampath National Laboratory Services, Milpark Hospital, Johannesburg, South Africa. ${ }^{19}$ Department of Surgery, School of Medicine, Washington University in Saint Louis, Saint Louis, MO, USA. ${ }^{20}$ Departments of Surgery and Anesthesiology, Division of Trauma and Surgical Critical Care, Vanderbilt University Medical Center, Nashville, TN, USA. ${ }^{21}$ Department of Surgery, University of Virginia Health System, Charlottesville, VA, USA. ${ }^{22}$ Departments of Medicine, Clinical Epidemiology and Biostatistics, and Pathology and Molecular Medicine, McMaster University, Hamilton, ON, Canada. ${ }^{23}$ Département d'Anesthésie-Réanimation, CHU Bichat

Claude-Bernard-HUPNVS, Assistance Publique-Hôpitaux de Paris, University Denis Diderot, Paris, France. ${ }^{24}$ Section of Critical Care Medicine and Section of Infectious Diseases, Department of Medicine, Medical Microbiology and Pharmacology/Therapeutics, University of Manitoba, Winnipeg, MB, Canada. ${ }^{25}$ Australia Pharmacy Department, Royal Brisbane and Womens' Hospital, Burns, Trauma, and Critical Care Research Centre, Australia School of Pharmacy, The University of Queensland, QLD, Brisbane, Australia. ${ }^{26}$ Department of Intensive Care, Erasme Hospital, Université libre de Bruxelles, Brussels, Belgium. ${ }^{27}$ Department of Internal Medicine, Division of Infectious Diseases, Akron General Medical Center, Northeast Ohio Medical University, Akron, OH, USA. ${ }^{28} \mathrm{Clinical}$ Microbiology and Infectious Diseases, School of Pathology, Faculty of Health Sciences, University of the Witwatersrand, Johannesburg, South Africa. ${ }^{29}$ Division of Infectious Diseases, Los Angeles County-University of Southern California (USC) Medical Center, Keck School of Medicine at USC, Los Angeles, CA, USA. ${ }^{30}$ Department of Infectious Diseases, Alfred Hospital, Melbourne, VIC, Australia. ${ }^{31}$ Department of Surgery, College of Health Sciences, Obafemi Awolowo University, Ile-lfe, Nigeria. ${ }^{32}$ Division of Clinical and Administrative Sciences, College of Pharmacy, Xavier University of Louisiana, New Orleans, LA, USA. ${ }^{33}$ Department of Medicine, Division of Infectious Diseases, University of South Carolina School of Medicine, Columbia, SC, USA. ${ }^{34}$ General Surgery, ULSS19 del Veneto, Adria Hospital, Adria, RO, Italy. ${ }^{35}$ Biomedical Research Center, Qatar University, Doha, Qatar. ${ }^{36}$ Department of Microbiology, Chitwan Medical College, and Department of Environmental and Preventive Medicine, Oita University, Oita, Japan. ${ }^{37}$ Centre for Neglected Tropical Diseases, Liverpool School of Tropical Medicine, University of Liverpool, and Mercy Hospital Research Laboratory, Njala University, Bo, Sierra Leone. ${ }^{38}$ Department of Surgery, University Hospital Center, Zagreb, Croatia. ${ }^{39}$ Trauma and Acute Care Surgery Unit, Hadassah Hebrew University Medical Center, Jerusalem, Israel. ${ }^{40}$ Department of Traumatology, John Hunter Hospital and University of Newcastle, Newcastle, NSW, Australia. ${ }^{41}$ Department of Surgery, Bizerte Hospital, Bizerte, Tunisia. ${ }^{42}$ Academic Department of Surgery, Queen Elizabeth Hospital, Birmingham, UK. ${ }^{43}$ Department of General Surgery, Hospital San Juan de Dios de La Serena, La Serena, Chile. ${ }^{44}$ Emergency Department, University of Leipzig, Leipzig, Germany. ${ }^{45}$ Department of Surgery, University of Colorado, Denver, CO, USA. ${ }^{46}$ Department of Surgery, Academic Medical Centre, Amsterdam, The Netherlands. ${ }^{47}$ Department of Pathology and Laboratory Medicine, VA Boston HealthCare System, and Department of Pathology and Laboratory Medicine, Boston University School of Medicine, Boston, MA, USA. ${ }^{48}$ Division of Acute Care Surgery, Department of Surgery, University of Michigan, Ann Arbor, MI, USA. ${ }^{49}$ Department of Surgery, Radboud University Nijmegen Medical Center, Nijmegen, The Netherlands. ${ }^{50}$ Department of Surgery, Hospital Clínico Universitario, Santiago de Compostela, Spain. ${ }^{51}$ Pharmacy Department, Alfred Health, Melbourne, VIC, Australia. ${ }^{52}$ Hospital Epidemiology and Infectious Diseases, Hospital Universitario Dr Jose Eleuterio Gonzalez, Monterrey, Mexico. ${ }^{53}$ Department of Pharmacology, Pushpagiri Institute of Medical Sciences and Research Centre, Thiruvalla, Kerala, India. ${ }^{54}$ Department of General Surgery, Kuala Krai Hospital, Kuala Krai, Kelantan, Malaysia. ${ }^{55}$ Department of Surgery and Obstetrics/Gynaecology, Regional Hospital, Limbe, Cameroon. ${ }^{56}$ Department of Mathematics, Imperial College London, London, UK. ${ }^{57}$ Department of Laparoscopic and Robotic Surgery, Colli-Monaldi Hospital, Naples, Italy. ${ }^{58}$ Department of Surgery, Tianjin Nankai Hospital, Nankai Clinical School of Medicine, Tianjin Medical University, Tianjin, China. ${ }^{59}$ Infectología Institucional SRL, Hospital Municipal Chivilcoy, Buenos Aires, Argentina. ${ }^{60}$ Department of Surgery, University Clinical Center of Tuzla, Tuzla, Bosnia and Herzegovina. ${ }^{61}$ Department General Surgery,
Kipshidze Central University Hospital, Tbilisi, Georgia. ${ }^{62}$ Department of Surgery, Quatre Villes Hospital, St Cloud, France. ${ }^{63}$ School of Pharmacy, Faculty of Medical Sciences, The University of the West Indies, St. Augustine, Eric Williams Medical Sciences Complex, Uriah Butler Highway, Champ Fleurs, Trinidad and Tobago. ${ }^{64}$ Division of Acute Care Surgery, Program in Trauma, $R$ Adams Cowley Shock Trauma Center, University of Maryland, Baltimore, MD, USA. ${ }^{65}$ Department of Surgical Sciences, Cannizzaro Hospital, University of Catania, Catania, Italy. ${ }^{66}$ Ifakara Health Institute, Dar es Salaam, Tanzania. ${ }^{67}$ Department of Surgery, Maggiore Hospital, Bologna, Italy. ${ }^{68}$ Center for Food Safety, Department of Food Science and Technology, University of Georgia, Griffin, GA, USA. ${ }^{69}$ School of Pharmacy and Biomedicine, Mongolian National University of Medical Sciences, Ulaanbaatar, Mongolia. ${ }^{70}$ Department of Surgery, University Hospital of Trauma, Tirana, Albania. ${ }^{71}$ Département d'Anesthésie-Réanimation, CHU Amiens-Picardie, and INSERM U1088, Université de Picardie Jules Verne, Amiens, France. ${ }^{72}$ Department of Surgery, Division of Burn, Critical Care, and Trauma Surgery (K.P.S., S.R.E.), Weill Cornell Medical College/New York-Presbyterian Hospital, New York, USA.

${ }^{73}$ Department of Medicine, Infectious Disease Division, King Fahad Medical City, Riyadh, Saudi Arabia. ${ }^{14}$ Department of Surgery, Pirogov Russian National Research Medical University, Moscow, Russian Federation. ${ }^{75}$ Sudan National Public Health Laboratory, Federal Ministry of Health, Khartoum, Sudan. ${ }^{76}$ Department of Surgery, Virginia Commonwealth University, Richmond, VA, USA. ${ }^{77}$ Department of Global Health and Population, Harvard T.H. Chan School of Public Health, Boston, MA, USA. ${ }^{78}$ Division of Trauma Surgery, Department of Surgery, School of Medical Sciences, University of Campinas (Unicamp), Campinas, SP, Brazil. ${ }^{79}$ Institut Pasteur, Abidjan, Ivory Coast. ${ }^{80} 6$ th Department of Internal Medicine, Hygeia General Hospital, Athens, Greece. ${ }^{81}$ Department of General Surgery, Mansoura Faculty of Medicine, Mansoura University, Mansoura, Egypt. ${ }^{82}$ 2nd Department of Surgery, Aretaieion University Hospital, National and Kapodistrian University of Athens, Athens, Greece. ${ }^{83}$ Department of Surgery, Hospital Universitário Terezinha de Jesus, Faculdade de Ciências Médicas e da Saúde de Juiz de Fora, Juiz de Fora, Brazil. ${ }^{84}$ Center for Global Health, Mito Kyodo General Hospital, University of Tsukuba, Mito, Ibaraki, Japan. ${ }^{85}$ Hospital Privado Centro Médico de Caracas and Hospital Vargas de Caracas, Caracas, Venezuela. ${ }^{86}$ Unit of Pharmacology, Faculty of Medicine and Defense Health, National Defence University of Malaysia, Kuala Lumpur, Malaysia. ${ }^{87}$ Institute of Hygiene,

Charité-Universitätsmedizin Berlin, Hindenburgdamm 27, 12203 Berlin, Germany. ${ }^{88}$ Department of General and Thoracic Surgery, University Hospital Giessen, Giessen, Germany. ${ }^{89}$ Orgamed Consulting, Bad Griesbach, Germany. ${ }^{90}$ Department of Surgery, St. Josef Hospital, Ruhr University Bochum, Bochum, Germany. ${ }^{91}$ Department of Surgery, Faculté de médecine, Université de Parakou, BP 123, Parakou, Bénin. ${ }^{92}$ Division of Trauma and Surgical Critical Care, Department of Surgery, Asan Medical Center, University of Ulsan College of Medicine, Seoul, Republic of Korea. ${ }^{93}$ Department of Visceral, Transplant and Thoracic Surgery, Innsbruck Medical University, Innsbruck, Austria. ${ }^{94}$ Department of Surgery Philadelphia VA Medical Center, Perelman School of Medicine, University of Pennsylvania, Philadelphia, PA, USA. ${ }^{95}$ Department of Microbiology, Gandhi Medical College, Bhopal, India. ${ }^{96} \mathrm{Clinic}$ for Emergency Surgery, Medical Faculty University of Belgrade, Belgrade, Serbia. ${ }^{97}$ Department of Anesthesiology and Intensive Care, Charité Universitätsmedizin Berlin, Campus Benjamin Franklin, Berlin, Germany. ${ }^{98} 3 \mathrm{rd}$ Department of General Surgery, Jagiellonian University Medical College, Krakow, Poland. ${ }^{99}$ Department of Pharmacology and Therapeutics, College of Health Sciences, Makerere University, Kampala, Uganda. ${ }^{100}$ Department of Surgery, Albert Einstein College of Medicine and Jacobi Medical Center, Bronx, NY, USA. ${ }^{101}$ Department of General Surgery, Division of Surgery, Rambam Health Care Campus, Haifa, Israel. ${ }^{102}$ Department of Emergency Surgery, City Hospital, Mozyr, Belarus. ${ }^{103}$ Department of Primary Care and Emergency Medicine, Kyoto University Graduate School of Medicine, Kyoto, Japan. ${ }^{104}$ Department of Surgery, The Brunei Cancer Centre, Jerudong Park, Brunei. ${ }^{105}$ Department of Surgery, Edendale Hospital, Pietermaritzburg, South Africa. ${ }^{106}$ School of Medicine, Western Sydney University, Campbelltown, NSW, Australia. ${ }^{107}$ Division of Acute Care Surgery and Surgical Critical Care, Department of Surgery, Los Angeles County and University of Southern California Medical Center, University of Southern California, Los Angeles, CA, USA. ${ }^{108}$ Department of General Surgery, Erzincan University, Faculty of Medicine, Erzincan, Turkey. ${ }^{109}$ Department of Pharmacy, Lebanese International University, Beirut, Lebanon. ${ }^{110}$ School of Life Science and The ithree Institute, University of Technology, Sydney, NSW, Australia.

${ }^{111}$ Department of Biomedical Sciences and Public Health, Unit of Hygiene, 
Preventive Medicine and Public Health, UNIVMP, Ancona, Italy.

${ }^{112}$ Department of Critical Care Medicine, Cliniques Universitaires Saint LuC, Université Catholique de Louvain (UCL), Brussels, Belgium. ${ }^{113}$ Department of Surgery, Division of Trauma, University of Arizona, Tucson, AZ, USA.

${ }^{114}$ Department of Surgery, Yonsei University College of Medicine, Seoul, South Korea. ${ }^{115}$ Texas Tech University Health Sciences Center School of Pharmacy, Abilene, TX, USA. ${ }^{116}$ Department of Anaesthesiology and Critical Care, Hôpital Nord, Assistance Publique-Hôpitaux de Marseille, Aix Marseille Université, Marseille, France. ${ }^{117}$ Abdominal Center, University Hospital Meilahti, Helsinki, Finland. ${ }^{118}$ Department of Surgery, Inling Hospital, Nanjing University School of Medicine, Nanjing, China. ${ }^{119}$ Division of Infectious Diseases, Division of Emergency Medicine, Washington University School of Medicine, St. Louis, MO, USA. ${ }^{120}$ Division of Infectious Diseases, Department of Clinical Pathology, Faculty of Medicine, University of Indonesia, Cipto Mangunkusumo General Hospital, Jakarta, Indonesia. ${ }^{121}$ Department for Traumatology and Orthopedic Surgery, Cologne Merheim Medical Center (CMMC), University of Witten/Herdecke (UW/H), Cologne, Germany. ${ }^{122}$ Health Research Program, Institute of Economic and Social Research, University of Zambia, Lusaka, Zambia. ${ }^{123}$ Multidisciplinary Intensive Care Research Organization (MICRO), Wellcome Trust-HRB Clinical Research, Department of Clinical Medicine, Trinity Centre for Health Sciences, St James' University Hospital, Dublin, Ireland. ${ }^{124}$ Department of Surgery, Post-Graduate Institute of Medical Sciences, Rohtak, India. ${ }^{125}$ Department of Clinical Pharmacology, School of Medicine, University of Botswana, Gaborone, Botswana.

${ }^{126}$ Department of Surgery, Radiology, University Hospital of the West Indies, Kingston, Jamaica. ${ }^{127}$ General Surgery Department, Centro Hospitalar de São João, Porto, Portugal. ${ }^{128}$ Department of Surgery, Emergency Hospital of Bucharest, Bucharest, Romania. ${ }^{129}$ Center of Anti-Infective Research and Development, Hartford, CT, USA. ${ }^{130}$ Department of Laboratory Medicine, Division of Clinical Microbiology, Karolinska University Hospital Huddinge, Stockholm, Sweden. ${ }^{131}$ Research Unit, Health Policy Consult, Weija, Accra, Ghana. ${ }^{132}$ Department of Surgery, King Abdullah University Hospital, Irbid, Jordan. ${ }^{133}$ Department of Surgery and Critical Care, Universidad del Valle, Fundación Valle del Lili, Cali, Colombia. ${ }^{134}$ Department of Surgery, Hassan II University Hospital, Medical School of Fez, Sidi Mohamed Benabdellah University, Fez, Morocco. ${ }^{135}$ Division of Emergency and Trauma Surgery, Ribeirão Preto Medical School, Ribeirão Preto, Brazil. ${ }^{136}$ Division of Surgery, Vittorio Emanuele Hospital, Catania, Italy. ${ }^{137}$ Department of General and Emergency Surgery, Riga East University Hospital 'Gailezers', Riga, Latvia. ${ }^{138}$ National Institute for Health Research, Health Protection Research Unit in Healthcare Associated Infections and Antimicrobial Resistance, Imperial College London, Hammersmith Campus, London, UK. ${ }^{139}$ Department of General Surgery, Jesenice General Hospital, Jesenice, Slovenia. ${ }^{140}$ Trauma and Acute Care Service, St Michael's Hospital, University of Toronto, Toronto, Canada. ${ }^{141}$ U.S. Naval Medical Research Unit No 6, Callao, Peru. ${ }^{142}$ General Surgery Department, Medical University, University Hospital St George, Plovdiv, Bulgaria. ${ }^{143}$ Intensive Care Department, Hospital Clínico San Carlos, Madrid, Spain. ${ }^{144} \|$ Cátedra de Clínica Quirúrgica, Hospital de Clínicas, Universidad Nacional de Asunción, San Lorenzo, Paraguay. ${ }^{145}$ Department of Surgery, Catholic University of Sacred Heart, Policlinico A Gemelli, Rome, Italy. ${ }^{146}$ Department of Surgery, Faculty of Medicine, Thammasat University Hospital, Thammasat University, Pathum Thani, Thailand. ${ }^{147}$ Department of General Surgery, Tan Tock Seng Hospital, Tan Tock Seng, Singapore, Singapore. ${ }^{148}$ Department of Gastrointestinal Surgery, Stavanger University Hospital, Stavanger, Department of Clinical Medicine, University of Bergen, Bergen, Norway. ${ }^{149}$ Department of Emergency Surgery and Critical Care, Centro Medico Imbanaco, Cali, Colombia. ${ }^{150}$ Department of Surgery, North Estonia Medical Center, Tallinn, Estonia. ${ }^{151}$ Surgery Health Care Group, Hull and East Yorkshire Hospitals NHS Trust, Hull, UK. ${ }^{152}$ APHP medical and infectious diseases ICU, Bichat Hospital, Paris, France. ${ }^{153}$ Institute of Microbiology, Biological and Environmental Sciences College, University San Francisco de Quito, Quito, Ecuador. ${ }^{154}$ Department of Molecular Biology, Tran Hung Dao Hospital, No 1, Tran Hung Dao Street, Hai Ba Trung Dist, Hanoi, Vietnam. ${ }^{155} 1$ st Department of Surgery - Department of Abdominal, Thoracic Surgery and Traumatology, General University Hospital, Prague, Czech Republic. ${ }^{156}$ Department of Surgery, Medical School University of Pécs, Pécs, Hungary. ${ }^{157}$ Nottingham Oesophago-Gastric Unit, Nottingham University Hospitals, Nottingham, UK. ${ }^{158}$ Department of Surgery, Sheri-Kashmir Institute of Medical Sciences, Srinagar, India. ${ }^{159}$ State Key Laboratory for Diagnosis and Treatment of Infectious Diseases, The First Affilliated Hospital, Zhejiang University, Zhejiang, China. ${ }^{160}$ Trauma and Emergency Surgery Department,
Chang Gung Memorial Hospital, Taoyuan City, Taiwan. ${ }^{161}$ Department of Surgery, MOSC Medical College Kolenchery, Cochin, India. ${ }^{162}$ Infection Control Unit, Angers University, CHU d'Angers, Angers, France. ${ }^{163}$ Division of Trauma and Surgical Critical Care, DeWitt Daughtry Family Department of Surgry, University of Miami, Miami, FL, USA. ${ }^{164}$ Anesthesia and Intensive Care Unit, AORN dei Colli Vincenzo Monaldi Hospital, Naples, Italy.

${ }^{165}$ Anesthesiology and Intensive Care Unit, Sant'Orsola University Hospital, Bologna, Italy. ${ }^{166}$ Infectious Diseases Unit, University of Genoa (DISSAL) and IRCCS San Martino-IST, Genoa, Italy. ${ }^{167}$ Infectious Diseases Unit, Department of Medical and Surgical Sciences, Sant' Orsola Hospital, University of Bologna, Bologna, Italy.

Received: 27 July 2017 Accepted: 27 July 2017

Published online: 02 August 2017

Reference

1. Sartelli $M$, et al. Antimicrobials: a global alliance for optimizing their rational use in intra-abdominal infections (AGORA). World J Emerg Surg. 2016;11:33. 